\title{
Pielonefrite cronica: una causa misconosciuta di perdita del trapianto di rene
}

\author{
Valeria Corradetti, Eleonora Pattonieri, Chiara Rocca, Teresa Valsania, Marilena Gregorini, Teresa Rampino, \\ Claudia Martinelli, Pasquale Esposito, Antonio Dal Canton
}

U.O. Nefrologia, Dialisi e Trapianto IRCCS Fondazione Policlinico San Matteo e Università degli Studi di Pavia, Pavia

\begin{abstract}
Chronic pyelonephritis: a misunderstood cause of renal allograft loss
Although urinary tract infections (UTI) are the most frequent infections in kidney transplant patients, there are no sufficient data about the prevalence of acute and chronic pyelonephritis and organ or patient survival.

We present the clinical case of a young woman with a kidney transplant admitted to our Nephrology Clinic because of an acute worsening of renal function and a history of recurrent UTI. The renal biopsy showed medullary tissue with a severe tubule-interstitial damage, suggestive for acute rejection, insomuch that steroids were prescribed. Due to the lack of any clinical improvement, a second biopsy was performed, showing a histological picture of chronic pyelonephritis with a severe and advanced damage. Subsequently, because of the severe renal failure the patient underwent peritoneal dialysis and transplant nephrectomy.

This clinical case is exemplificative of the possible adverse outcomes of recurrent UTI that might lead in a short time to organ failure, highlighting the importance of a definitive histologic diagnosis.
\end{abstract}

Keywords: Chronic pyelonephritis, Recurrent/relapsing urinary tract infections, Kidney transplant

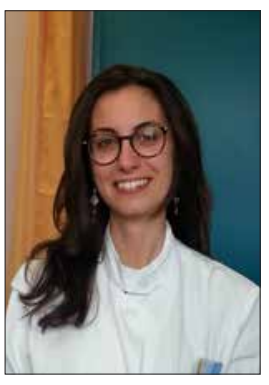

Valeria Corradetti

\section{Introduzione}

Le infezioni delle vie urinarie hanno un'alta incidenza nei pazienti trapiantati di rene e si complicano frequentemente con pielonefrite (1).

Anche se i dati in letteratura sono scarsi, la maggior parte degli Autori concorda sul fatto che episodi di pielonefrite acuta siano associati ad aumentate morbilità e mortalità (2-5).

La pielonefrite acuta nel trapianto può decorrere asintomatica, poiché il rene trapiantato è denervato (6). Generalmente per la diagnosi ci si può avvalere dei seguenti criteri (7): batteriuria significativa, con eventuale urocoltura positi-

Accepted: February 6, 2015

Published online: May 7, 2015

Indirizzo per la corrispondenza:

Dr. Pasquale Esposito

U. O. Nefrologia, Dialisi e Trapianto

IRCCS Fondazione Policlinico San Matteo

Piazzale Golgi 19

27100 Pavia

pasqualeesposito@hotmail.com va, febbre $>38^{\circ} \mathrm{C}$ e/o dolore in corrispondenza della sede del graft e/o riduzione acuta della funzionalità renale. La pielonefrite cronica si presenta, invece, con un quadro clinico più insidioso, manifestandosi soprattutto con i sintomi e i segni di danno tubulo-interstiziale cronico, mentre raramente si rinvengono in anamnesi significativi e ricorrenti episodi di pielonefrite acuta. La mancanza di dati istologici raccolti sistematicamente comporta probabilmente una sottostima degli episodi di pielonefrite acuta e non permette di porre diagnosi precoce di pielonefrite cronica; inoltre alcuni reperti morfologici sono analoghi a quelli di rigetto acuto, per cui talora la diagnosi differenziale può risultare difficile.

\section{Caso clinico}

Una donna di 33 anni giungeva nel nostro ambulatorio per peggioramento progressivo della funzione renale nell'ultimo anno.

La paziente, monorene dalla nascita, aveva iniziato il trattamento emodialitico extracorporeo nel 2005 a seguito di una verosimile pielonefrite. In anamnesi presentava un singolo ricovero per pielonefrite e negava infezioni recidivanti delle vie urinarie, mentre la cistografia retrograda e minzionale non evidenziava un reflusso vescico-ureterale.

$\mathrm{Nel} 2006$ era stata sottoposta a trapianto di rene da do- 
natore deceduto con regolare decorso post-operatorio e aveva ricevuto terapia di induzione con basiliximab e steroide e mantenimento con tacrolimus, micofenolato mofetile e steroide (poi sospeso al primo anno post-trapianto). Nel periodo di follow-up sono da segnalare una malattia citomegalica secondaria, trattata con valganciclovir, e la positivizzazione di Polyomavirus BK su siero e urine a febbraio 2009, che avevano richiesto la riduzione della terapia immunosoppressiva. A fine 2009, riscontro bioptico di rigetto acuto cellulare concomitante con un peggioramento acuto della creatininemia trattato con 3 boli da $500 \mathrm{mg}$ di metilprednisolone e.v., a seguito dei quali i valori di creatinina si erano stabilizzati su circa $2.5 \mathrm{mg} / \mathrm{dL}$. Durante tutto il periodo di follow-up si segnalano infezioni delle vie urinarie ricorrenti e recidivanti da Escherichia Coli, alcune delle quali da ceppi ESBL produttori, sempre trattate secondo antibiogramma e mai sospette per pielonefrite. Nel 2013 la paziente si trasferiva presso il nostro centro trapianti, documentando un peggioramento della funzione renale, con valori di creatinina saliti in circa due mesi fino a $4 \mathrm{mg} / \mathrm{dL}$. La terapia immunosoppressiva consisteva in tacrolimus e micofenolato mofetile. Agli ematochimici gli indici di flogosi risultavano negativi, così come la conta leucocitaria, l'ecografia renale mostrava scarsa differenziazione cortico-midollare, assenza di dilatazione delle vie urinarie e resistenze intraparenchimali del $70 \%$ e il sedimento urinario mostrava numerosi batteri, $10 \mathrm{WBC} /$ campo ad alto ingrandimento e numerose cellule di sfaldamento di alte e basse vie urinarie. In quell'occasione l'urinocoltura risultava negativa, così come risultavano negativi la ricerca di PVBK-DNA su siero e urine e gli anticorpi donatore-specifici (DSA).

In considerazione del rapido rialzo della creatininemia e della storia di riduzione della terapia immunosoppressiva, si decideva di eseguire una biopsia renale. II quadro istologico mostrava solo tessuto midollare con denso infiltrato infiammatorio composto da elementi linfoidi di piccole dimensioni costituito prevalentemente da cellule CD3+ e in parte da elementi CD20+, plasmacellule politipiche, numerosi neutrofili e rari eosinofili. II tessuto non presentava glomeruli e nel poco tessuto risparmiato si riconoscevano rari tubuli atrofici e fibrosi interstiziale marcata, mentre risultava negativa la ricerca immunoistochimica per l'antigene SV40 del Polyomavirus BK (Fig. 1). Poiché il referto era di non univoca interpretazione, nel sospetto di una nefrite interstiziale attribuibile a un rigetto cellulare acuto, si somministrava steroide a boli endovenosi e poi per via orale; in considerazione del fatto che la biopsia, priva di glomeruli, era poco diagnostica e che la paziente non presentava una risposta soddisfacente alla terapia steroidea, che comunque non si era associata alla comparsa di infezioni, si decideva di eseguire una nuova biopsia. Quest'ultimo campione mostrava 30 glomeruli per sezione istologica, il $50 \%$ dei quali in totale involuzione scleroialina; i restanti glomeruli risultavano contratti con capsule di Bowmann ispessite e sclerosi mesangiale di grado variabile da lieve-moderato a marcato

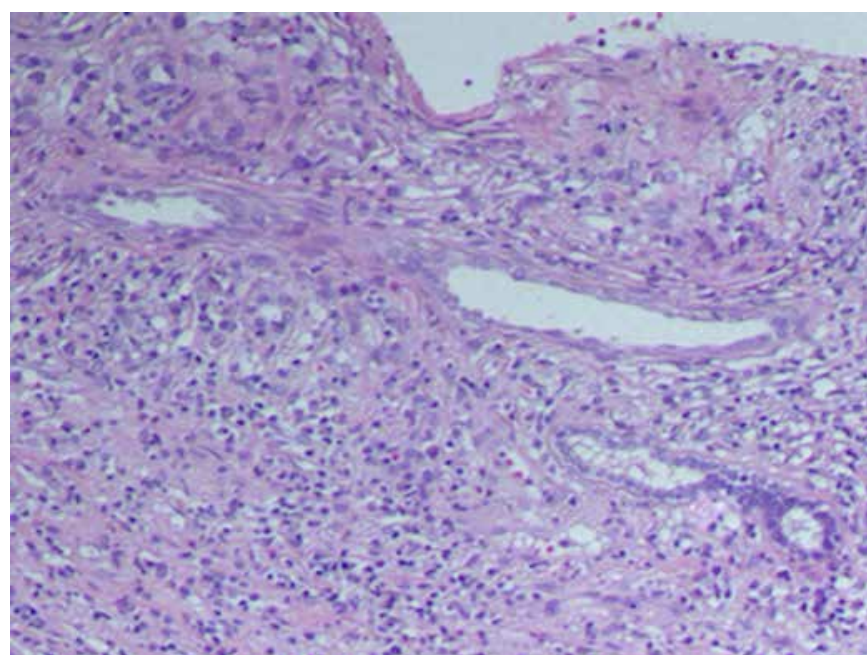

Fig. 1 - Infiltrato infiammatorio denso e diffuso costituito da linfociti CD3+ e CD20+, plasmacellule politipiche, numerosi neutrofili e rari eosinofili (ematossilina eosina 10X).

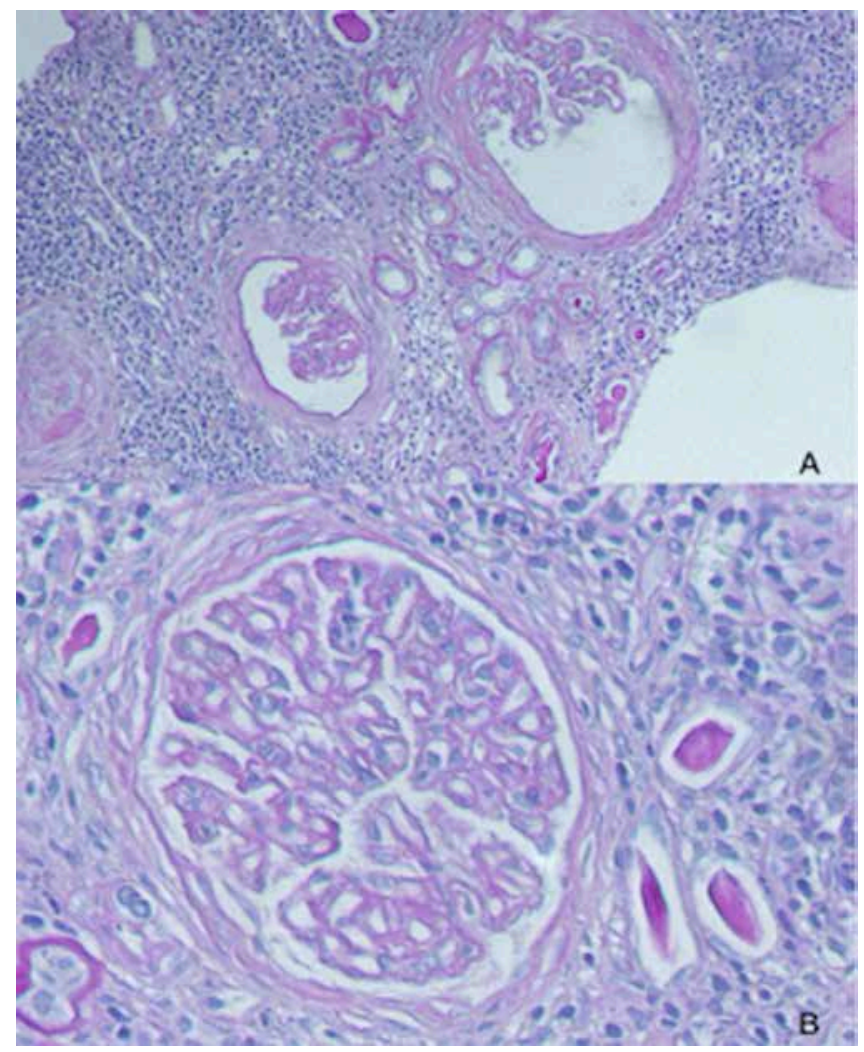

Fig. 2 - Glomeruli contratti con capsule di Bowmann ispessite e sclerosi mesangiale di grado variabile fino a completa involuzione sclero-ialina (A: PAS 10X; B: PAS 20X).

(Fig. 2); i tubuli mostravano modificazioni atrofiche e tiroidizzazione (Fig. $3 \mathrm{~A}$ ), con marcato ispessimento associato delle membrane basali tubulari ed epitelio assottigliato (Fig. 3B), con interstizio costituito da estesi infiltrati densi di elementi linfoidi CD3+ e CD20+, istiociti, plasmacellule 


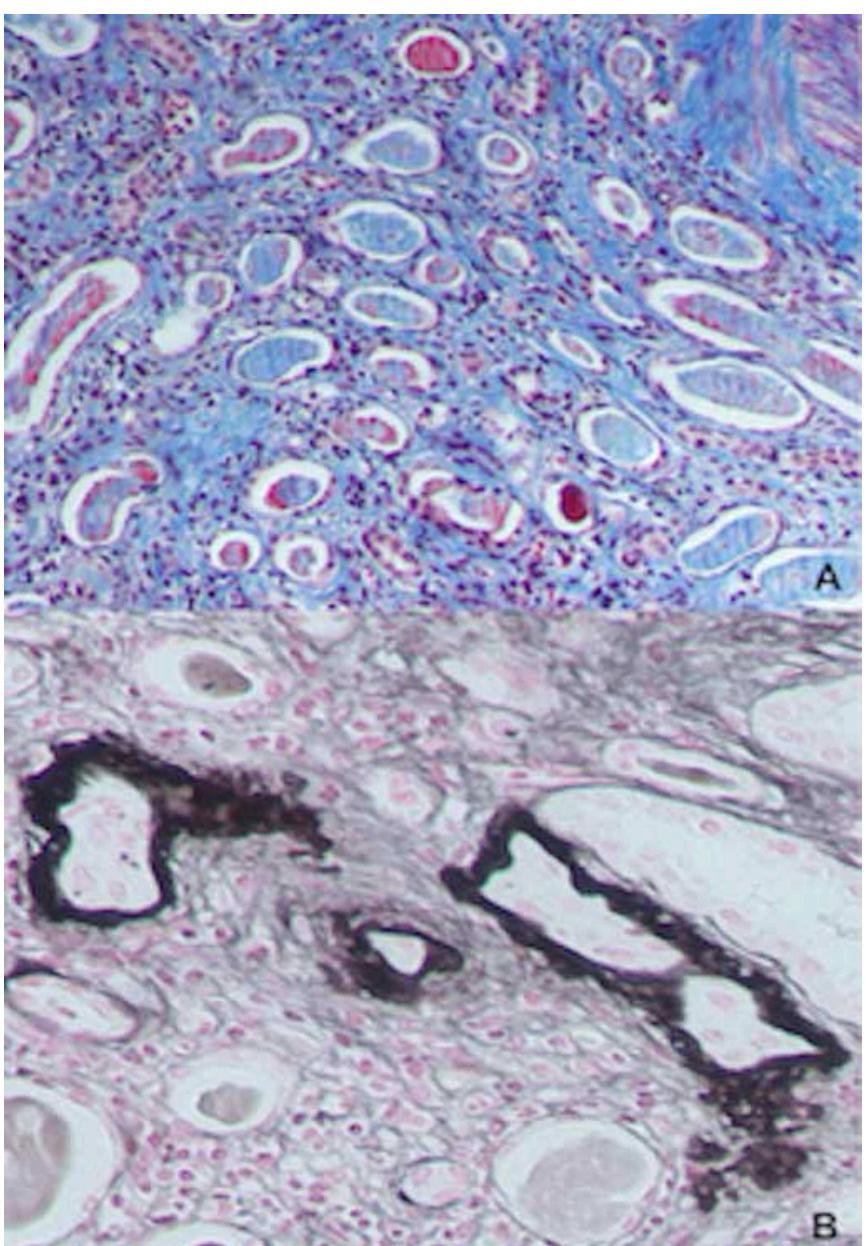

Fig. 3 - Atrofia tubulare e fibrosi interstiziale. A) Fibrosi interstiziale diffusa con tiroidizzazione. L'epitelio tubulare appare assottigliato e i lumi tubulari sono pieni di materiale amorfo (Masson 10X). B) Marcato ispessimento delle membrane basali tubulari (PASM 20X).

e significativa quota di granulociti neutrofili. I vasi arteriosi presentavano ispessimento fibromuscolare parietale, mentre risultava negativa l'immunoistochimica per $\mathrm{C} 4 \mathrm{~d}$. Questo campione bioptico, pertanto, mostrava reperti adeguati per una diagnosi di malattia tubulo-interstiziale tipo pielonefrite cronica con danno glomerulare secondario.

Nel frattempo, nel corso dei 15 giorni necessari per ottenere i due referti istologici, la funzione renale era peggiorata ulteriormente con valori fino a $5 \mathrm{mg} / \mathrm{dL}$, pari a un GFR di $15 \mathrm{~mL} / \mathrm{min} / 1.73 \mathrm{mq}$. Alla luce del referto bioptico suggestivo per un danno renale ormai avanzato, la paziente veniva, quindi, avviata al trattamento dialitico peritoneale.

La paziente successivamente presentava un buono stato di salute, mentre la diuresi si riduceva progressivamente fino all'anuria in circa 3 mesi. La terapia immunosoppressiva veniva progressivamente ridotta, eliminando dapprima il micofenolato mofetile e, poi, lo steroide e mantenendo il tacrolimus a basse dosi.
Circa 8 mesi dopo l'inizio della dialisi peritoneale, la paziente presentava febbre e dolore in corrispondenza della sede del graft. Le urinocolture davano ripetutamente esito negativo, mentre un'ecografia mostrava un rene trapiantato con corticale assottigliata e idronefrosi di I grado. Pertanto, la sintomatologia della paziente era attribuita a una sindrome infiammatoria legata alla presenza del rene trapiantato e alla riduzione dell'immunosoppressione e si decideva di procedere all'espianto. Dopo l'intervento, svoltosi senza complicanze, la paziente si manteneva in buone condizioni e attualmente sta eseguendo gli esami per essere nuovamente inserita in lista attiva per trapianto renale.

\section{Discussione}

Le IVU rappresentano la complicanza infettiva più frequente nei pazienti trapiantati di rene e sono di particolare importanza in quanto possono influenzare la sopravvivenza dell'organo e/o del paziente $(8,9)$.

Questo vale anche per la batteriuria asintomatica, entità molto frequente nel post-trapianto e spesso sottovalutata, che rappresenta un fattore di rischio per lo sviluppo di complicanze più severe tra cui anche le pielonefriti (10).

Nonostante la potenziale importanza delle IVU nel determinare la prognosi del trapianto, non sono disponibili dati epidemiologici consistenti, atti a definire la prevalenza e l'incidenza di IVU complicate, in particolar modo di pielonefrite acuta e cronica. I pazienti trapiantati di rene, oltre alle problematiche legate all'immunosoppressione, presentano alcuni peculiari fattori predisponenti allo sviluppo delle pielonefriti (8): stasi urinaria derivante da possibili stenosi dell'anastomosi uretero-vescicale o dell'uretere, anamnesi di malattia da reflusso vescico-ureterale nei reni nativi come potenziale sorgente di batteri anche per l'eventuale stasi post-chirurgica, anamnesi di calcolosi a stampo, persistenza di stent ureterali e/o di catetere vescicale e alta prevalenza di diabete; a questi fattori si aggiunge l'anatomia post-trapianto che, pur in presenza di una plastica anti-reflusso, spesso evidenzia la libera risalita di urina dalla vescica.

La diagnosi di pielonefrite acuta avviene generalmente con criteri clinici (11) e strumentali. Tra questi, l'esame gold standard è rappresentato dalla TC con mezzo di contrasto, il cui uso risulta limitato dal potenziale effetto negativo sullafunzione renale alterata (12). In alternativa, l'ecografia con mezzo di contrasto si sta imponendo nella pratica clinica sia per la facilità di esecuzione e per l'assenza di tossicità che per le sue elevate sensibilità e specificità (12), mentre si ricorre molto raramente alla biopsia renale.

L'unico studio che presenta dati morfologici si basa sulla conferma istologica in ogni caso di riduzione acuta della funzione renale; in quella popolazione, il $25 \%$ dei pazienti mostrava reperti compatibili con pielonefrite acuta, circa un quarto aveva colture negative e il 5\% non aveva alcun segno clinico di infezione delle vie urinarie (6). 
Il nostro caso mette in risalto anche la difficoltà nel porre diagnosi differenziale tra pielonefrite e rigetto acuto.

La prima biopsia ha mostrato, infatti, soltanto del tessuto midollare con reperti che non hanno permesso di esprimere una chiara diagnosi differenziale tra pielonefrite e rigetto acuto.

Infatti, entrambi i quadri patologici presentano un marcato coinvolgimento tubulo-interstiziale e l'infiltrato infiammatorio può comprendere tutte le classi di leucociti. Nel rigetto acuto è costituito soprattutto da linfociti $T$ e macrofagi con evidenza di tubulite e, secondo il grado di danno, si possono osservare anche distruzione della membrana basale tubulare e invasione dei tubuli da parte di cellule infiammatorie con scomparsa dell'abituale organizzazione del tessuto (13, 14). Nella pielonefrite acuta si possono trovare soprattutto neutrofili accompagnati da plasmacellule distribuiti in microascessi che distruggono il tessuto; dal momento che la patogenesi di tale malattia è la risalita del patogeno attraverso le vie urinarie, il dato più saliente risulta la presenza di cast di neutrofili, soprattutto nella midollare. Nel caso della pielonefrite cronica, l'infiltrato è costituito da neutrofili e linfociti $T$ e B e comuni sono i cast cellulari di leucociti e la presenza di follicoli linfoidi (14). Nella nostra prima biopsia era presente solamente l'infiltrato infiammatorio con caratteristiche comuni a rigetto acuto e pielonefrite, ma non erano presenti due dati importanti, come la tubulite, che avrebbe fatto deporre per un caso di rigetto, o i cast cellulari di neutrofili, reperto rilevante nelle pielonefriti. Dal momento che erano presenti elementi a favore di un evento acuto, abbiamo intrapreso un trattamento immunosoppressivo, a seguito del quale abbiamo ripetuto la biopsia, così da poter arrivare a una diagnosi di certezza. La seconda biopsia ci ha mostrato, invece, tutte le caratteristiche tipiche della pielonefrite cronica. Infatti, è risultata evidente l'atrofia tubulare con tiroidizzazione diffusa circondata da tubuli con membrane basali ispessite ed epitelio assottigliato, l'interstizio risultava espanso e, dove non occupato da infiltrato infiammatorio, si mostrava occupato da materiale fibrotico; i glomeruli erano obsoleti, alcuni con ispessimento della capsula di Bowmann e ialinizzazione più o meno marcata; queste caratteristiche sono tipiche di un danno renale a partenza dal tubulo interstizio come la pielonefrite.

Non è, comunque, escludibile che la perdita della funzione renale nella nostra paziente sia stata dovuta alla concomitanza di diversi fattori (per esempio, pielonefrite, tossicità da $\mathrm{CNI}$ e rigetto cellulare). Infatti, mentre l'eziologia virale è stata esclusa con tecniche di immunoistochimica per l'antigene SV40 del Polyomavirus BK, alcune porzioni della biopsia mostravano tutti i reperti caratteristici di un danno cronico nel rene trapiantato. L'estensione e l'entità del danno, unite alla giovane età della paziente, ci hanno poi convinto a intraprendere rapidamente il trattamento dialitico.

\section{Conclusioni}

Questo caso clinico risulta emblematico del problema rivestito dalla potenziale evoluzione negativa delle IVU ricorrenti e recidivanti in una paziente in cui gli episodi di pielonefrite sono risultati asintomatici e hanno condotto in un tempo relativamente breve alla perdita dell'organo. Una diagnosi istologica definitiva in pazienti con IVU recidivanti, anche non accompagnate da un peggioramento della funzione renale, permetterebbe di caratterizzare meglio l'entità delle pielonefriti asintomatiche indirizzando a una terapia adeguata e più precoce.

\section{Disclosures}

Financial support: No financial support was received for this submission.

Conflict of interest: The authors have no conflict of interest.

\section{Bibliografia}

1. Schmaldienst $S$, Dittrich $E$, Horl WH. Urinary tract infections after renal transplantation. Curr Opin Urol 2002;12:125-30.

2. Munoz P. Management of urinary tract infections and lymphocele in renal transplant recipients. Clin Infect Dis 2001; 33(Suppl. 1):S53-7.

3. Pellé G, Vimont S, Levy PP, et al. Acute pyelonephritis represents a risk factor impairing long-term kidney graft function. Am J Transplant 2007;7:899-907.

4. Abbott KC, Swanson SJ, Richter ER, et al. Late urinary tract infection after renal transplantation in the United States. Am J Kidney Dis 2004;44:353-62.

5. Giral M, Pascuariello G, Karam G, et al. Acute graft pyelonephritis and long-term kidney allograft outcome. Kidney Int 2002;61:1880-6.

6. Varma PP, Jairam A, Hooda AK, Badwal S. Renal outcome in biopsy proven cases of graft pyelonephritis. Indian J Nephrol 2014;24(3):161-5.

7. Gołębiewska J, Dębska-ślizień A, Zadrożny D, Rutkowski B. Acute graft pyelonephritis during the first year after renal transplantation. Transplant Proc 2014;46(8):2743-7.

8. De Souza RM, Olsburgh J. Urinary tract infection in the renal transplant patient. Nat Clin Pract Nephrol 2008;4:252-64.

9. Chuang $P$, Parikh CR, Langone A. Urinary tract infection after renal transplantation: a retrospective review at two US transplant centers. Clin Transplant 2005;19:230-5.

10. Singh R, Geerlings SE, Bemelman FJ. Asymptomatic bacteriuria and urinary tract infections among renal allograft recipients. Curr Opin Infect Dis 2015;28(1):112-6.

11. El Amari EB, Hadaya K, Buhler L, et al. Outcome of treated and untreated asymptomatic bacteriuria in renal transplant recipients. Nephrol Dial Transplant 2011;26:4109-14.

12. Granata A, Andrulli S, Fiorini F, et al. Diagnosis of acute pyelonephritis by contrast-enhanced ultrasonography in kidney transplant patients. Nephrol Dial Transplant 2011;26: 715-20.

13. Racusen LC, Solez K, Colvin RB, et al. The Banff 97 working classification of renal allograft pathology. Kidney Int. 1999 Feb;55(2):713-23.

14. Weiss M, Liapis H, Tomaszewski JE, Arend LJ. Chronic Pyelonephritis in Jennette JC, Olson JL, Schwartz MM, Silva FG, ed Heptinstall's Pathology of the Kidney $6^{\text {th }}$ edition, Lippincott Williams \& Wilkins, 2007;1003-5. 


\section{TEST DI VERIFICA}

1. Quale dei seguenti segni esclude la presenza di pielonefrite acuta?
a) Assenza di febbre
b) Dolore in sede di trapianto
c) Urocoltura negativa
d) Nessuna delle precedenti

2. Quali strumenti possono aiutare a porre diagnosi di pielonefrite cronica?
a) Solo segni clinici
b) È necessaria la TC
c) Anche la biopsia renale può essere utile
d) $a+b$

3. Quale dei seguenti è un aspetto istologico tipico delle pielonefriti?
a) Glomerulite
b) Tubulite
c) Ispessimento delle pareti vascolari
d) Cast di neutrofili intratubulari

Le risposte corrette alle domande sono pubblicate su questo numero alla pagina 98. 